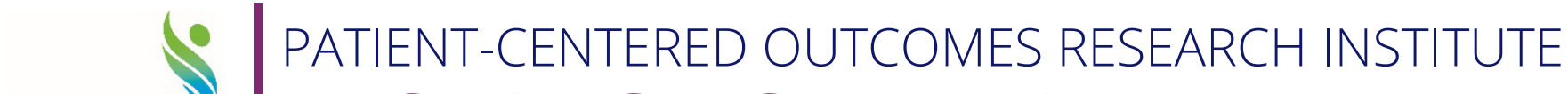 pcori). RESEARCH SUMMARY
}

\section{Comparing Two Ways to Manage Symptoms for Patients Who Have Chronic Migraine and Frequent Medication Use -- The MOTS Trial}

Principal investigator

Todd J. Schwedt, MD, MS
Organization

Mayo Clinic Arizona

\section{What was the research about?}

Chronic migraine is when people have headaches 15 or more days per month, with migraines for at least 8 of those days. Patients can take medicines, such as non-steroidal anti-inflammatory medicines and triptans, to relieve symptoms. But about half of people with chronic migraine use these medicines more often than recommended because of the pain and disability the headaches cause. When these medicines are taken too often for more than three months, it's called medication overuse. Medication overuse can lead to more frequent headaches, migraine attacks, and other serious side effects.

In this study, the research team compared two approaches to treat adult patients with chronic migraine and medication overuse. In both, patients took a medicine to prevent migraines. Then patients either:

- Stopped taking the overused medicine and switched to a different type of medicine up to two days per week to relieve symptoms, or

- Continued taking the overused medicine on as many days as needed to relieve symptoms.

\section{What were the results?}

After 12 weeks, the two approaches worked about the same to reduce the number of days with a migraine.
Patients who stopped taking the overused medicine had about 9.3 migraine days per month compared with 9.1 days for patients who continued it. The research team's statistical analysis showed that staying on the overused medicine wasn't worse for controlling symptoms than switching medicines.

But patients who stopped the overused medicine took medicine to treat symptoms less often than patients who continued it. They were also less likely to overuse migraine medicines.

The two approaches didn't differ in:

- Patients' quality of life

- Whether migraines made it hard to do daily tasks or caused disability

- Adverse events, such as high blood pressure, fatigue, or stomach pain

- Whether patients remained in the study

\section{Who was in the study?}

The study included 720 adult patients with chronic migraine and medication overuse. Of these, 75 percent were White, 6 percent were Black, and 1 percent were Asian; 13 percent were Hispanic. The average age was 44 , and 87 percent were women. Patients received care at one of 34 headache, 
neurology, or primary care clinics across the United States.

\section{What did the research team do?}

The research team assigned patients by chance to one of the two treatment approaches. They surveyed

patients at the start of the study and again 9-12 weeks later.

Patients kept a diary for weeks 1-12 and other 4-week periods over one year. They recorded how often they had headaches, how intense they were, and how long they lasted. Patients also recorded whether they used medicine to treat symptoms.

Patients and headache specialists helped plan and conduct the study.

\section{What were the limits of the study?}

The study didn't look at how different types of medicines prevent migraines or relieve symptoms.

Future research could compare how different types of medicines improve migraines and medication overuse.

\section{How can people use the results?}

Patients and their doctors can use the results when treating chronic migraine and medication overuse.

To learn more about this project, visit www.pcori.org/Schwedt395. 